\title{
APROPIACIÓN Y USO DE SALAS DE ELABORACIÓN POR PARTE DE AGRICULTORES FAMILIARES DE LA PROVINCIA DE MISIONES, ARGENTINA $^{1}$
}

\author{
María Inés Mathot y Rebolé ${ }^{2}$ \\ Fernando Pablo Landini ${ }^{3}$
}

\section{RESUMEN}

En el presente artículo se analiza la construcción, apropiación y uso de salas de elaboración por parte de agricultores familiares de la provincia de Misiones (Argentina) en el contexto de un proyecto financiado por el Estado. La investigación fue cuali-cuantitativa. Se realizaron 24 entrevistas semi-estructuradas a agricultores familiares participantes del proyecto y a los tres técnicos implicados. Para el análisis, se construyeron tres ejes de estudio: percepción de los agricultores sobre la necesidad de la sala y de su uso; obstáculos y recursos de los beneficiarios para afrontar la construcción; y disponibilidad de recursos externos. Independientemente de si la sala había sido terminada, en general las familias encontraron usos para ellas, tanto productivos como de almacenamiento. Los agricultores familiares mencionaron múltiples obstáculos para la construcción, destacándose el económico. Sin embargo, también evidenciaron haber tenido recursos personales para afrontar estos obstáculos. Finalmente, se destaca que el acompañamiento técnico fue un recurso valioso, que tuvo un rol fundamental en la finalización de las salas.

Palabras clave: Agricultura familiar, extensión rural, psicología rural, salas de agroindustria.

\section{APROPRIAÇÃO EUSO DE SALAS DE ELABORAÇÃO POR PARTE DE AGRICULTORES FAMILIARES NA PROVÍNCIA DE MISIONES (ARGENTINA).}

\section{RESUMO}

Neste artigo analisa-se a construção, apropriação e utilização de salas de elaboração por parte de agricultores familiares da província de Misiones (Argentina) no contexto de um projeto apoiado pelo Estado. A pesquisa foi quali-quantitativa. Foram realizadas 24 entrevistas semi-estruturadas a agricultores familiares que participavam do projeto e aos três técnicos implicados. Para a análise, construíramse três eixos de estudo: percepção dos beneficiários da necessidade da sala e seu uso; obstáculos e recursos dos beneficiários para enfrentar a construção; e

${ }^{1}$ La presente investigación fue financiada por el Comité Ejecutivo de Desarrollo e Innovación Tecnológica (CEDIT) de la provincia de Misiones, Argentina, por medio de una beca otorgada a la primera autora

${ }^{2}$ Graduada en Psicología (UBA). Maestranda en Desarrollo Rural (UNaM).Profesora Adjunta de la Universidad de la Cuenca del Plata (UCP). E-mail: marinemathot@gmail.com.

${ }^{3}$ Graduado en Psicología (UBA). Magíster en Desarrollo Rural (UPM). Dr. en Psicología (UBA). Investigador Adjunto del Consejo Nacional de Investigaciones Científicas y Técnicas (CONICET). Profesor Asociado de la UCP. Profesor Invitado de la Universidad de Morón. Email: landini_fer@hotmail.com. 
disponibilidade de recursos externos. Independentemente de a sala haver sido terminada, em geral as famílias encontraram usos para elas, tanto produtivos quanto de armazenamento. Os agricultores familiares mencionaram numerosos obstáculos, sendo o mais importante o econômico. Não obstante, eles também mostraram ter recursos pessoais para enfrentar estes obstáculos. Finalmente, destaca-se que o acompanhamento técnico foi um recurso valioso, jogando um papel fundamental na finalização das salas.

Palavras-chave: agricultura familiar, extensão rural, psicologia rural, salas de agroindústria.

\title{
APPROPRATION AND USE OF PROCESSING FACILITIES BY FAMILY FARMERS IN THE PROVINCE OF MISIONES ARGENTINA
}

\begin{abstract}
The present paper analyses the construction, appropriation and use of processing facilities by family farmers in the province of Misiones (Argentina) in the context of a state funded project. The research was both qualitative and quantitative. 24 interviews were conducted engaging semi-structured family farmers project participants and three technicians. To the analysis, three lines of study were developed: farmers perception on the need of the processing facilities and its use; obstacles and resources that beneficiaries faced through construction; and availability of external resources. Regardless of whether the processing facilities had been completed, the families mainly found uses for them, both productive and storage. Family farmers mentioned many obstacles through construction, highlighting the economic issue. However, they also showed personal resources to face these obstacles. Finally, we emphasize that the technical support was a valuable resource, which played a key role in the completion of the processing facilities.
\end{abstract}

Keywords: family farming, processing facilities, rural extension, rural psychology.

\section{INTRODUCCIÓN}

Las ferias francas ('feiras livres' en portugués) son un fenómeno económico que logra crear una conexión directa entre los agricultores familiares y los compradores (MOREL; REZENDE; SETTE, 2015), evitando intermediarios, razón por la cual en diferentes países latinoamericanos se han constituido en una estrategia de trabajo prioritaria en el contexto de la extensión rural. En la provincia de Misiones (Argentina), las ferias francas surgen a partir del año 1995 como alternativa económica en un momento de crisis para el sector. Desde ese año, ellas continúan y se fortalecen, siempre con la premisa de sostener un espíritu de intercambio justo entre agricultores familiares y consumidores.

Desde la década del 90, las ferias francas han despertado el interés de diferentes disciplinas, dentro de las cuales se destacan ciencias sociales como la antropología, la economía y la sociología. Si bien a primera vista podría pensarse que las ferias generarían más interés en disciplinas relacionadas con la producción y la ingeniería en alimentos, el carácter familiar de los agricultores que participan en ellas y la importancia que tienen las relaciones sociales en su dinámica explican esta 
situación, que nos habla de su complejidad.

Los emprendimientos de feria tienen valor como espacios económicos (SIQUEIRA et al., 2013) pero también como espacios de socialización y apoyo mutuo (BUSSO, 2011; BURNETT, 2014). En un relevamiento realizado en Argentina, Golsberg et al. (2010) contabilizaban 144 ferias francas. Desde una visión económica, las ferias francas han sido abordadas como "mercados locales", aunque aún sin la capacidad de abastecer plenamente las necesidades alimentarias de la población local (ROSENFELD; KRIEGER; CAZZANIGA, 2005). Por otro lado, hay autores que sostienen que, al surgir en Argentina en un momento de crisis (RODRÍGUEZ et al., 2010), las ferias francas han contribuido a la consolidación de un imaginario social que asocia ruralidad y pobreza, particularmente por implementarse de manera contemporánea con un programa del Estado destinado a paliar la pobreza rural, el Programa Social Agropecuario (SCHVORER, 2007).

A su vez, autores como García (2008) y García y Wahren (2005) entienden a las ferias francas misioneras como espacios de "resistencia campesina". También existen estudios que destacan el valor histórico del surgimiento y desarrollo de las ferias francas en la provincia en respuesta a una crisis económica (BUSSO, 2011). En esta línea, puede afirmarse que si bien las ferias francas han brindado alternativas económicas a los agricultores familiares, su influencia es más amplia, ya que los espacios de feria significan nuevas prácticas en cuanto a la forma de producir y comercializar (CARBALLO, 2002).

Desde su surgimiento en el año 1995, las ferias francas se fueron multiplicando en la provincia de Misiones y en el país. Así, se hizo cada vez más evidente la importancia de la problemática de la higiene y de la manipulación de los alimentos, particularmente de aquellos productos elaborados por las familias como quesos y conservas, ya que la salud de la población consumidora estaba en juego. De hecho, existe un conjunto de trabajos que abordan la cuestión de las ferias francas en general y de las salas de elaboración de los agricultores familiares en particular desde una perspectiva más ligada con la ingeniería en alimentos. Dentro de ellos pueden mencionarse el análisis realizado por Fernández y Picciarelli (2012) sobre las salas de elaboración de agricultores familiares dedicados a la producción de quesos que luego son comercializados en la ciudad de Posadas; o estudios acerca de los criterios de evaluación bromatológica y nutricional de productos preelaborados (DI PIETRO et al., 2004; MARZOCCA; MARUCCI; ÁLVAREZ, 2004; MICCIO et al., 2011; OLIVERA-CARRION, 2011). En esta línea, también pueden incluirse trabajos que abordan la disposición operativa y las buenas prácticas para la manipulación de alimentos en una sala de elaboración en la pequeña agricultura familiar (ALIAGA, 2011).

En Misiones, en el año 1998 comienza a discutirse la necesidad de generar salas de agroindustria para que los agricultores familiares tengan la posibilidad de elaborar sus productos en condiciones satisfactorias de higiene y salubridad. No obstante, es recién en el año 2007 que se aprueba el proyecto "Salas de Elaboración de Productos Artesanales en el Marco de la Seguridad Alimentaria", el cual es objeto de la investigación que aquí se presenta. El proyecto fue presentado al gobierno de manera conjunta por la Asociación UNESCO Corrientes, Caritas Posadas, el equipo Justicia, Paz e Integridad de la Creación (JUPIC) y la Asociación de Productores que agrupa a los agricultores que participaban de la feria franca de la ciudad de Posadas. La financiación provino del Programa de Salud Alimentaria del Ministerio de Desarrollo Social de la Nación, mientras que las instituciones que lo impulsaron aportaron el seguimiento técnico.

El proyecto partió de un diagnóstico participativo, en el contexto del cual los agricultores decidieron priorizar (1) la ampliación de los espacios para procesar la 
producción y (2) el saneamiento ambiental del sistema de la vivienda con construcción de baños y mejoramiento de los existentes, dadas las condiciones precarias en las cuales estaban elaborando los alimentos que luego llevaban a la venta en la feria. En este contexto, el proyecto se dividió en tres componentes: sala de elaboración, equipamiento básico de cocina, y capacitación y seguimiento técnico. A nivel más operativo, el proyecto preveía "readecuar mamposterías, pisos, instalaciones y equipamiento" del lugar donde se realizaran las elaboraciones. No obstante, en el proyecto no se explicitaban los materiales con los cuales debía armarse cada sala de elaboración. Sí se aclaraba que el proyecto buscaba que los agricultores pudieran contar con espacios apropiados de cocina, depósito y baño (separados de la vida doméstica) con el fin de cuidar la cadena de seguridad sanitaria de los productos destinados al consumo y, especialmente, a la venta en la feria.

Cuando se pone en marcha el proyecto, el equipo técnico de las instituciones impulsoras, en acuerdo con la organización de productores de la feria franca Posadas, selecciona 24 grupos de agricultores familiares que participaban de la feria, cada uno compuesto por cinco familias. La expectativa era que cada grupo seleccionara el predio de una de las familias para construir allí una sala de elaboración de agroindustria, que sería utilizada de manera conjunta. En términos generales, los agricultores familiares en cuyos predios finalmente se construyeron las salas contaban con un promedio de entre 10 y 20 hectáreas, se dedicaban a la producción de huerta (lechuga, tomate, zanahoria, acelga, rabanito y zapallo, entre otros), tanto para el autoconsumo como para la venta de excedentes. Esto se complementaba con la cría de animales vacunos, porcinos y de aves de corral, según la disponibilidad de mano de obra familiar. En algunos casos, a esto se agregaba la producción de yerba mate.

En un relevamiento preliminar realizado en el contexto de esta investigación entre los años 2011 y 2012, se observó que los grupos de agricultores familiares sólo habían permanecido unidos durante un período limitado de tiempo, y que las salas de elaboración construidas, así como el equipamiento respectivo, había quedado a disposición de las familias dueñas de los predios, con lo que el esperado uso colectivo había devenido en un uso individual.

En su mayoría las salas habían sido construidas con mampostería, incluyendo revestimiento de azulejos en la zona de la pared donde estaba la mesada de trabajo, que era de acero inoxidable. Los pisos eran de cemento alisado y los techos de chapa de zinc. No obstante, en numerosos casos las salas no estaban finalizadas, faltaban elementos de terminación en varios de sus componentes y su uso no necesariamente se ajustaba a lo planificado.

Revisando la experiencia latinoamericana, los problemas enfrentados por el proyecto "Salas de Elaboración de Productos Artesanales en el Marco de la Seguridad Alimentaria" no resultan inesperados. De hecho, autores como Boas y Goldey, (2005) en Brasil y Landini (2007) en Argentina, a partir de diferentes estudios territoriales, muestran la dificultad del trabajo asociativo entre productores. Más aún, en un artículo reciente, Landini (2016) señala que las dificultades a nivel de trabajo grupal o asociativo constituyen el problema más mencionado por los extensionistas rurales en América Latina, lo que destaca aún más la importancia de esta problemática.

A la vez, la segunda dificultad que se observa en el caso del proyecto de Salas de Elaboración se relaciona con la participación, el compromiso y la apropiación del proyecto por parte de los beneficiarios, lo que se expresó como falta de terminación y de uso de las salas. Nuevamente, Boas y Goldey (2005) señalan que en diferentes proyectos de desarrollo rural se han observado dificultades para 
lograr una participación comprometida por parte de los beneficiarios. En este sentido, múltiples autores han señalado que esto puede deberse a que los proyectos no contaron con una participación real de los beneficiarios en su diseño (SARAIVA; CALLOU, 2009) o a que las acciones propuestas no correspondían con las expectativas de los agricultores (TAVEIRA; OLIVEIRA, 2005; FREITAS et al., 2008).

Ahora bien, sin asumir que las características del caso descripto son generalizables, sí se observa que en éste aparecen de manera transversal problemáticas relevantes para el trabajo de los extensionistas rurales latinoamericanos, por lo que su análisis puede permitir extraer aprendizajes de interés para la labor de los asesores técnicos. En concreto, en el presente trabajo se busca comprender la apropiación subjetiva y el uso que los agricultores familiares beneficiarios del proyecto hicieron de las salas de elaboración, haciendo énfasis en la incidencia de la necesidad percibida de contar con las salas y la disponibilidad de recursos (propios y externos) para llevar adelante el proyecto. Así, en el contexto de un abordaje orientado al actor (LONG, 2007), se retoma la importancia del nivel de determinación psicosocial de la agencia humana (LANDINI et al., 2014a; LANDINI et al., 2014b; LANDINI, 2015), luego del reconocimiento que hace recientemente Long (2015) respecto a la importancia de enriquecer el abordaje orientado al actor desde la psicología. Así, en este trabajo se retoman conceptos tradicionalmente utilizados por la psicología como el de 'necesidad percibida' y de 'percepción de recursos' (este último en el sentido de creencia de que se dispone de herramientas para enfrentar de manera satisfactoria diferentes problemáticas o desafíos), con el fin de comprender el uso y apropiación subjetiva que los beneficiarios del proyecto han hecho de las salas de elaboración.

\section{METODOLOGÍA}

Para alcanzar los objetivos propuestos se realizó una investigación cualicuantitativa, utilizando como metodología el estudio de caso (MONTERO; LEÓN, 2005). La investigación focalizó en la toma y análisis de entrevistas semiestructuradas. Se realizaron entrevistas a los 24agricultores familiares en cuyos predios se acordó la construcción de las salas de elaboración y a los tres técnicos que participaron del proyecto. Las entrevistas se realizaron entre julio de 2011 y septiembre de 2012.

Las entrevistas a los beneficiarios se realizaron en las salas. El registro se hizo de forma escrita. En ellas se abordaron los siguientes ejes:

- Infraestructura y características de la unidad productiva.

- Historia de la forma de producir previa al proyecto y modo de acceso al mismo.

- Evaluación personal de la necesidad que tenían de contar o no con una sala de elaboración.

- Identificación de necesidades actuales, desde su propia perspectiva.

- Obstáculos y facilitadores para la puesta en marcha de la construcción de la sala.

- Nivel de construcción y utilización actual de la sala.

- Existencia o no de acompañamiento institucional, de qué tipo y en qué momentos.

Las entrevistas realizadas a técnicos fueron grabadas. Las mismas incluyeron preguntas relativas a los siguientes ejes:

- Cuándo se inició el proyecto.

- Proceso de implementación. Su rol como técnicos y la actitud de los agricultores familiares respecto del proyecto. 
- Facilitadores y obstáculos que surgieron.

- Rol del Estado.

- Seguimiento del proyecto.

En un primer momento, la información descriptiva de las entrevistas realizadas a los agricultores fue sistematizada en una en planilla de cálculo. Allí se volcaron datos sobre la unidad de producción, la infraestructura del domicilio, los servicios disponibles, el estado de la sala y el equipamiento entregado. En segundo lugar, en base a las respuestas dadas por los agricultores familiares, se construyeron las siguientes áreas temáticas o ejes de análisis:

1. Percepción de los beneficiarios sobre la necesidad de la sala y su uso actual. Para trabajar esta área temática se utilizaron las respuestas a las preguntas en las cuales se abordó la evaluación de los beneficiarios respecto a la necesidad de contar o no con una sala de elaboración, y el nivel de construcción de las salas y su uso actual. Para analizar las respuestas se utilizaron diferentes postulados de la teoría de la acción razonada (ROTH, 2008). En primer lugar, se asume que las personas generan acciones para mejorar las circunstancias en las que se encuentran. Así, mientras mayor incomodidad y malestar perciban en relación a su situación actual, mayor predisposición tendrán para generar acciones que permitan modificar aquello que les desagrada. De esta forma, se esperaría que los beneficiarios que estuvieran elaborando en situaciones más incómodas (es decir, que sintieran mayor necesidad de contar con una sala de elaboración), tendrían una actitud más positiva hacia su construcción y uso. No obstante, también hay que tener en cuenta el rol que juega la evaluación que hagan los agricultores de los beneficios y perjuicios que podría generar la construcción y el uso de la sala. En segundo lugar, para comprender la posibilidad de una conducta también hay que tener en cuenta las normas subjetivas, entendidas como la influencia (las opiniones) de su entorno social respecto de dicha conducta. Así, desde la Teoría de la Acción Razonada se considera que la intención de realizar una conducta (en este caso construir y utilizar la sala) estaría dada por la actitud positiva frente a dicha conducta y por las normas subjetivas (u opinión) de los pares frente a ella.

En el contexto de este eje, se analiza la relación entre la percepción de que se necesitaba o no la sala, y su nivel de terminación y tipo de uso (productivo o no productivo). En línea con los desarrollos teóricos precedentes, se esperaría que quienes expresaron necesidad de la sala la hubieran terminado y la estuvieran usando para fines productivos. En contraste, sería de esperar que aquellos que no hubieran expresado la necesidad de una sala no la hubieran terminado o, en caso de haberlo hecho, que no la estuvieran usando para los fines previstos (es decir, productivos).

2. Obstáculos y recursos propios percibidos por los agricultores para afrontar la construcción y el uso de las salas. Para estructurar este eje se siguió el enfoque de la teoría de la acción razonada (ROTH, 2008). Y las propuestas del modelo transteórico de Prochaska para pensar los procesos de cambio (PROCHASKA; NORCROSS; DICLEMENTE, 1994). A partir de esto, se hipotetizó que los productores que percibieran mayores obstáculos, desafíos y necesidades relacionados directa o indirectamente con la construcción y uso de las salas, y aquellos que evaluaran que contaban con menos recursos y capacidades para afrontarlos, tendrían menos probabilidad de haberlas concluido. Y a la inversa, quienes percibieran menores obstáculos y mayor disponibilidad de recursos para hacerles frente, tendrían mayores probabilidades de haberlas terminado.

Partiendo de estas ideas se subdividió el eje de análisis en percepción de obstáculos y percepción de capacidades personales para afrontarlos. Para esto se utilizaron las respuestas a las preguntas de las entrevistas donde se abordaron 
obstáculos y facilitadores internos con los que se encontraron en el proceso, otras necesidades percibidas antes de la construcción y en la actualidad, y cómo sería la práctica actual sin la sala de elaboración.

Con el fin de facilitar el análisis, se construyeron dos escalas. Para la escala de obstáculos, se asignó un punto a cada obstáculo y uno a cada necesidad mencionada en las entrevistas. Para aquellos que tuvieron un puntaje de hasta dos se consideró que, desde su perspectiva, evaluaban que los obstáculos y/o desafíos a afrontar eran "pocos". Para quienes obtuvieron de tres a cinco puntos que éstos eran "bastantes" y para quienes obtuvieron seis o más que los obstáculos y/o desafíos eran "muchos".

Para la escala de recursos y capacidades propias para enfrentar los obstáculos y/o desafíos se otorgó un punto a cada facilitador mencionado (por ejemplo tener materiales de construcción disponibles en el predio como madera, o presencia de personas con experiencia en construcción en la zona, entre otros) y un punto a quienes mencionaron apoyo de su familia o apoyo de su comunidad (en tanto opinión positiva) respecto de la construcción de la sala (dos puntos si el apoyo era de ambos). De esta manera, se consideró que quienes tenían hasta dos puntos se percibían a sí mismos con "pocos" recursos y capacidades para afrontar los obstáculos y desafíos que tenían que enfrentar, quienes tenían entre tres y cinco que contaban con "ciertos" recursos, y quienes obtuvieron seis o más que disponían de "muchos" recursos para hacerlo.

Cabe destacar que la construcción de este tipo de escalas constituye una estrategia operativa para comparar entre los diferentes casos y poder contrastar de manera ordenada los resultados con las hipótesis propuestas. En este sentido, no se argumenta que exista una razón intrínseca o de alguna manera 'objetiva' o 'verdadera' para asignar los puntajes y establecer las líneas de corte entre niveles como se hizo. No obstante, sí se sostiene que la propuesta constituye una estrategia útil para ordenar, sistematizar y exponer los resultados obtenidos, a diferencia de lo que podría haber sido un simple análisis descriptivo de cada uno de los casos.

3. Recursos externos percibidos por los agricultores para afrontar la construcción y el uso de las salas. Con el fin de abordar la temática se utilizaron los ejes de las entrevistas referidos a la historia de cómo se implementó el proyecto y el acompañamiento de técnicos e instituciones para su elaboración y posterior puesta en marcha, en tanto son aquellos recursos que el productor no posee pero a los cuales puede recurrir o ha recurrido en algún momento. Aquí también se incluyen recursos externos como los que brinda la comunidad en tanto ayuda, como ser: asistir con mano de obra o materiales, entre otros. A nivel de hipótesis, sería esperable que el apoyo externo sea predictor de finalización y uso de las salas.

A los fines de analizar los datos y poder comparar entre los diferentes agricultores familiares entrevistados, las respuestas a las preguntas se volcaron en una escala donde se reflejase el apoyo externo recibido, desde el punto de vista de los propios productores. Para esto se asignó un punto a cada institución (estatal o privada) mencionada como proveedora de algún tipo de ayuda, un punto si tuvo acompañamiento técnico antes del proyecto, y uno más si también lo tuvo durante el proyecto. En base a esto se construyó una variable ordinal considerando que quienes tuvieron un punto o menos no contaron con "ningún" recurso externo, quienes tuvieron entre dos y cuatro contaron con "algunos" recursos, y quienes tuvieron cinco o más que contaron con "muchos" recursos externos.

Entrevistas a técnicos. Como se indicó previamente, también se realizaron entrevistas a los tres técnicos que participaron del proyecto, las cuales fueron grabadas y transcriptas. Para el análisis de la información en el apartado de Resultados se sistematizan las respuestas siguiendo dos ejes: perspectiva de los 
beneficiarios en relación a la necesidad (o no) de una sala de elaboración, y obstáculos identificados para la implementación del proyecto y la construcción de las salas. En este proceso, también aparece un eje transversal, que es la concepción de su propio rol como asesores técnicos.

\section{RESULTADOS}

\subsection{Percepción de los beneficiarios sobre la necesidad de la sala y su uso actual}

En base al relevamiento realizado se pudo observar que de las 24 salas, 12estaban terminadas, 11 no habían sido finalizadas y en un caso no se había comenzado con la construcción. Sin embargo, más allá de la terminación se vio que, aunque el objetivo del proyecto era que se hiciera uso productivo de las salas, se encontraron 11 en uso productivo (familiar o comunitario), ocho como lugar de almacenamiento (familiar y/o comunitario), cuatro sin uso y una de ellas no construida. Es decir que, independientemente de si la sala había sido terminada, las familias encontraron usos para las salas, si bien no necesariamente para elaborar productos, sí relacionados tangencialmente con la producción (almacenamiento). De esta manera, se encontraba una situación no prevista: las salas en su mayoría sí recibían un uso aunque no fuera productivo, lo que evidenciaba la flexibilidad de las familias para apropiarse de las propuestas y recursos que recibían según sus propios intereses y lógicas (LANDINI, 2011).

Partiendo de quelas necesidades en relación a la alimentación de las familias de los entrevistados estaban resueltas, se pensó que aquellos que se encontraban ante una mayor incomodidad a la hora de dar a sus productos valor agregado (mermeladas, conservas, panificados y chacinados, entre otros), tendrían una más elevada predisposición para la finalización y el uso productivo de las salas. Así, también estarían más predispuestos quienes se hallaran en situación de mayor necesidad, en tanto precariedad para su subsistencia, en pos de optimizar sus ingresos económicos, ya que las salas serían un recurso para mejorar sus condiciones de producción y potenciar sus ingresos.

En primer lugar, si bien veinte de los 24agricultores familiaresentrevistados dijeron que al iniciar el proyecto pensaban que era necesario un lugar exclusivo de elaboración, también plantearon que las condiciones de trabajo previas no eran extremadamente incómodas. Es decir, no se percibía como una situación imperiosa de ser cambiada. Antes que se les propusieran las salas,16agricultores utilizaban la cocina familiar para la elaboración, 6 la cocina familiar y el exterior, y 2 no estaban elaborando. Siguiendo la Teoría de la Acción Razonada, se había pensado que el grado de incomodidad sería predictor de construcción. Sin embargo, al ser la cocina familiar el lugar de elaboración, este era un lugar vivido como incluido en las lógicas de la casa, más frecuentemente relacionadas con las prácticas de la mujer. Por lo dicho en las entrevistas, la elaboración de productos, antes de las salas, tenía una relación más estrecha con los alimentos que se hacían también para el consumo familiar. Por ejemplo, si se cocinaban conservas para la familia se hacía un extra para la comercialización. Dice una entrevistada: "vos hacías mermelada, lo que salía, y lo que quedaba llevabas a la feria. Ahora acá hago sólo para vender". Así, a partir de las salas, la tarea de elaborar para la venta se ve como una actividad específica, aparte de las cotidianas, como un momento especialmente destinado a la producción. Es decir, lo que formaba parte de una rutina más doméstica, familiar, cobra estatus de producción comercializable. Se observa entonces que la incomodidad de las condiciones de producción previas no puede ser pensada como 
un indicador de la construcción de las salas o de su uso productivo, ya que en general puede verse que a priori no existía percepción de incomodidad, lo que podría sugerir que la construcción se relacionó más con una influencia externa.

Cuando se preguntó "¿Cómo sería su práctica actual sin la sala de elaboración?", sólo 5 entrevistados plantearon que la hubieran hecho, y 10 que hubieran seguido como antes (sin cambio en las prácticas de elaboración), mientras que el resto sugirió que tal vez hubieran dejado la elaboración. Es decir, 19 de los 24 piensan que no hubieran innovado en sus prácticas e incluso 9 de ellos que tal vez las hubieran abandonado.

Si bien desde un principio parecería que los agricultores familiares no consideraban como una necesidad contar con salas de elaboración, sí reconocieron luego que tener un espacio exclusivo para la producción los había beneficiado. En las entrevistas a los agricultores, cuando se preguntó acerca de su práctica actual en las salas, 11 plantearon que ahora su práctica productiva de elaboración era más cómoda, 1 que producía más volumen y2 que realizaban mejores prácticas. Los 10 casos restantes no perciben cambios significativos en sus prácticas, de ellos 4 no hacen uso de la sala y 1 no inició la construcción. Es decir, 14 de los 24 casos consideran que la sala constituyó una innovación práctica positiva, aún cuando originalmente no sentían incomodidad o malestar con sus prácticas de elaboración.

\subsection{Obstáculos y recursos propios percibidos para afrontar el cambio}

Respecto de este eje y utilizando las categorizaciones indicadas en la metodología, se observa que 15 de los 24agricultores entrevistados tuvieron que enfrentarse a entre "bastantes" y "muchos" obstáculos para poder construir las salas. El obstáculo percibido como principal fue el económico (la falta de dinero en efectivo para comprar lo que hiciera falta) siguiéndole, con menor frecuencia, los problemas de accesibilidad (fundamentalmente el estado de los caminos para llegar al predio), y por último conseguir mano de obra especializada para trabajar en la construcción. Analizando estos resultados, se observa que de estos 15 agricultores sólo uno no pudo construir la sala, con lo cual la existencia de obstáculos y dificultades no parece ser el factor determinante para avanzar con la edificación, ya que en tal caso tendrían que haberse construido muchas menos. Teniendo en cuenta que 23 de las salas fueron construidas total o parcialmente, puede afirmarse que la existencia de obstáculos y necesidades percibidas no parece ser determinante de la construcción, por lo que habría también que evaluar su relación con la evaluación o percepción de disponibilidad de recursos o capacidades propias para afrontar los obstáculos.

Analizando esta variable, se observa que 13 de los agricultores familiares entrevistados contaban con ciertos recursos y capacidades propias para afrontar los obstáculos existentes, mientras que 7 disponían de "muchos" recursos. Es significativo que de estos veinte casos que se perciben con recursos están los 19agricultores familiaresque hacen algún tipo de uso de la sala de elaboración, lo que sugiere que la percepción de recursos y capacidades propias se relacionaría con el uso actual de las salas.

Por su parte, en relación a los recursos y capacidades de los agricultores, se encontró que en 9 de las 12salas terminadas se habían utilizado estrategias que combinaban el uso de diferentes recursos para la lograr la edificación. Por ejemplo, disponibilidad de mano de obra familiar, más existencia de materiales para la construcción accesibles en el predio (madera, piedra, etc.), por mencionar una de las combinaciones más frecuente. De esto se sigue que, primero, la disponibilidad de recursos y capacidades propios es fundamental para la construcción de las salas; y segundo, que esta disponibilidad no suele restringirse a una sola alternativa. Como 
argumento adicional, se menciona que en ninguno de los 13 casos en los cuales la construcción no había sido finalizada se mencionó el uso de estrategias que combinaran múltiples recursos para la edificación.

Por último, se destaca que en todos los casos los agricultores entrevistados coinciden en que el gran facilitador fue contar con dinero en efectivo recibido como subsidio para poder iniciar la construcción.

\subsection{Recursos externos percibidos para afrontar el cambio, más allá del subsidio recibido}

Teniendo en cuenta los diferentes niveles de recursos externos disponibles establecidos en la metodología, se observa que 6 no contaron con ninguno, 11 con algunos y 7 con muchos. Se destaca aquí que los criterios de clasificación de los casos, como fue sugerido previamente, no toman en cuenta como apoyo externo el hecho de haber recibido el subsidio para la construcción de las salas, ya que se trata de una variable que no permite establecer diferencias, al ser igual para todos.

Analizando los resultados, se observa que en los 7casos en los cuales se recibió mucho apoyo, la sala ha sido terminada completamente. A la vez, los 11 que han contado con algunos recursos externos han terminado total o parcialmente la sala. En la misma línea, estos 18 productores hacen algún uso de su sala.

Reflexionando sobre estos resultados, puede concluirse la pertinencia de la hipótesis propuesta, de que a mayor disponibilidad de recursos externos, mayor grado de terminación de las salas. A la vez, a la vista de los datos, también puede argumentarse que la disponibilidad de apoyo se relaciona con el hecho de darle un uso a las salas, ya que entre los 6 agricultores familiares que indicaron no haber contacto con ningún apoyo se encuentra el que no construyó la sala y los 4 que no hacen uso de ella.

\subsection{Aportes de los técnicos}

Con respecto a la percepción de los beneficiarios en relación a la necesidad (o no) de una sala de elaboración y a su uso, los técnicos afirman en las entrevistas que los agricultores familiares no siempre visualizan determinadas problemáticas, por lo que no parecen tener claridad respecto de la necesidad de contar con salas de elaboración. Uno de los técnicos entrevistados trae un argumento frecuente de los agricultores: "bueno, si esto nosotros hemos comido por años, y nunca nos enfermamos, ¿porqué ahora se van a enfermar [los compradores]". Y luego agrega "[su posición] era defensiva. Defender sus costumbres, sus formas de hacer las cosas". En este sentido, puede observarse que aquí se enfrentan diferentes lógicas, la de los técnicos y la de los agricultores familiares. Plantea otro técnico "y desde los agricultores familiares también falta otro camino por recorrer [...] tener conciencia que un alimento puede lastimar [en tanto comprometer la salud], al margen de la buena voluntad". En este contexto, los técnicos se asignan a sí mismos un rol: el de "sensibilizar" a los agricultores familiares, para que tomen conciencia de la importancia de las salas: "diseñar y ejecutar estrategias para sensibilizar sobre la problemática, porque a veces la gente se acostumbra a trabajar así". No obstante, esta necesidad de sensibilizar no debe ser pensada únicamente como un proceso unidireccional, sino más bien como "espacios de trabajo y reflexión que contribuían al abordaje de esa problemática".

En paralelo, esta preocupación se ramifica hacia otra. Los técnicos también argumentan que resulta necesario hacerles seguimiento a los productores para que utilicen los recursos del subsidio que reciben para el destino establecido, es decirla 
construcción de las salas. Las prácticas a revisar refieren a que un productor que, según plantean los técnicos entrevistados, concibe como innecesario producir los dulces para la venta separados de los alimentos que él mismo consumirá. En relación a no percibir esta necesidad es que el productor podría querer invertir el dinero destinado a comprar materiales para la sala con otros fines. A nivel práctico, esto implicaba "hacer un monitoreo de que el dinero que se le entregue para la compra de materiales esté, sea utilizado para justamente el objetivo, no se desvíen para otros lados".

En relación al eje de obstáculos identificados para la implementación del proyecto y la construcción de las salas, los técnicos afirman que uno de los principales fue la dificultad de los agricultores familiarespara entregar las rendiciones del dinero invertido, ya que ellos no están familiarizados con el llenado de formularios o con cuestiones contables. Uno de los técnicos explica: "lleva su tiempo explicarle a los agricultores familiares, porque por ahí no están acostumbrados a manejar las cuestiones de facturación, es un tema complejo". Así, esto agrega otro elemento al rol del asesor técnico: aportar en la gestión y rendición de recursos, ya que de otra forma los agricultores familiares no podrían acceder a proyectos complejos.

En relación al acompañamiento a los agricultores en la implementación del proyecto, en las entrevistas con los técnicos quedó de manifiesto que si bien se hizo bastante seguimiento al inicio, con el paso del tiempo los técnicos fueron reduciendo su participación. Esto se observa cuando afirman que "yo ya después no participé porque estuve en otra tarea", "después gestionamos otras cosas y eso siguió su curso". Ciertamente, esta situación podría pensarse como un problema, ya que tal vez un mayor seguimiento hubiera aumentado el porcentaje de salas terminadas. No obstante, también sería posible pensar que la falta de seguimiento del proyecto fue una estrategia de los mismos técnicos para no poner en evidencia que los proyectos no llegaban a concretarse totalmente.

\section{CONSIDERACIONES FINALES}

Se parte de la idea de que la organización social es el resultado de interacciones, negociaciones y luchas que se dan entre diferentes actores. Estas luchas transforman las relaciones que están en interjuego, determinándose mutuamente. Esto define la capacidad de agencia humana para la construcción y reproducción del tejido social (LONG, 2007). Las salas de elaboración leídas como espacios de puja social implicaron la concreción de una lucha mucho más amplia. En un primer momento del proyecto de salas, más ligado a los años '98 y '99, el Movimiento Agrario Misionero (MAM) y la Asociación de Ferias que agrupaba a los feriantes de la ciudad de Posadas trabajaron con los agricultores familiares de Misiones con metodologías participativas buscando identificar necesidades. En ese momento el Estado (estructurado según una lógica neoliberal) no contemplaba a los agricultores familiares como actores sociales ni agentes de intervención más allá de situaciones de pobreza.

A partir de la aparición de las ferias francas (entre otros fenómenos como los foros de agricultura familiar, etc.) y la llegada del slogan 'de la chacra a su mesa' (frase que se utilizó en los inicios de las ferias francas), los agricultores familiares comienzan a visibilizarse. Para los consumidores urbanos éstos tienen una imagen positiva y una alta carga afectiva (CARBALLO et al., 2008), y para parte del Estado comienzan a ser considerados un sector de interés.

En relación a las salas, el proyecto se materializa a partir del año 2007. Cuando finalizó la presente investigación en el año 2012, el nivel de construcción fue 
de 12 finalizadas, 11 parcialmente terminadas y una que no había sido iniciada. Si los parámetros para medir el éxito de este proyecto fueran los de finalizar la sala de elaboración, el $50 \%$ de los beneficiarios lograron el fin. Este es el tipo de evaluación que suele hacer el Estado al finalizar un proyecto, desconociendo las situaciones puntuales de los beneficiarios. Esto se enmarca en una lógica de planificación lineal verticalista, dentro de la cual se ponen en marcha proyectos y se espera que los agricultores familiares se adecuen. Esto es muy difícil, ya que los requisitos que pide el Estado son difíciles de alcanzar por los agricultores familiares beneficiarios, ya que los requisitos establecidos tienden a pensarse a partir de un tipo ideal de productor, que no coincide con el que se encuentra en la región. Además el Estado no solo evalúa al productor, el técnico también se ve atrapado entre responder a lo que se espera desde lo institucional o dar una respuesta real a los agricultores familiares. Para saldar este espacio, los técnicos buscan por un lado "sensibilizar" a los agricultores familiares para que perciban como necesidad algo que no siempre sienten así. Como los técnicos son conscientes de esto, se observan desvíos, como adaptar lo más posible el proyecto que se ofrece armado a la situación real del productor. Esta negociación se da de forma implícita o explícita.

En el caso del proyecto de Salas de Elaboración se observa que 19 salas tienen algún tipo de uso (almacenamiento, por ejemplo) contra cuatro que están sin uso y una sin construir. Esto permite pensar que existía una cierta necesidad por cubrir. En algunos casos coincidió con la de los productores (o éstos se 'sensibilizaron' de la necesidad en el proceso). En otros las salas se utilizaron para cubrir otras necesidades, con lo cual fueron igualmente útiles. Aparejado a esto, la falta de seguimiento puede leerse también como una estrategia para no poner en evidencia estas adaptaciones de los proyectos. En este sentido puede pensarse que, al inicio del proyecto, técnicos y agricultores familiares hacen un pacto tácito de no puesta en evidencia mutua. Lo interesante de esto es que muestra la tirantez que viven los técnicos extensionistas a la hora de mediar entre Estado y agricultores familiares, la cual podría resolverse con proyectos que surjan de forma más dialógica, es decir, respondiendo a las políticas que propone el Estado pero dando a los proyectos un margen de maniobra que permita adaptar la ejecución de recursos a la situación local. De alguna forma, legalizar y sistematizar lo que ya ocurre.

Cuando se preguntó a los productores cómo sería su práctica actual sin la sala de elaboración, sólo cinco plantearon que la hubieran hecho. Sin embargo, 19 fueron construidas total o parcialmente y tienen algún tipo de uso valorado por los agricultores. Esto sirve para pensar que no hay que caer en la falacia de que el productor es el único que posee conocimiento válido para su vida productiva, los técnicos aportan propuestas interesantes y útiles. Las salas son ejemplo de ello, que como proyecto impactaron en las prácticas de los agricultores familiares, generando cambios. Entonces, para lograr propuestas más adaptadas, es importante la implicación de técnicos y agricultores familiares en situaciones participativas, pero sabiendo que es un espacio de construcción en el cual las dos partes buscan influenciarse y donde deberán negociar para salir ambas beneficiadas (LANDINI; MURTAGH; LACANNA, 2009).

Los resultados muestran que la existencia de recursos externos que apoyaron a los productores resultó fundamental. A la vez, el acompañamiento técnico durante y después de otorgar el recurso también resultó sumamente valioso. Los casos en los que existió acompañamiento (aunque no fuera de la institución original) tuvieron mejores resultados. Si bien desde un principio queda claro quelos agricultores familiares no consideraban que contar con salas de elaboración fuera una necesidad, a posteriori reconocieron que tener un espacio exclusivo para la producción los había beneficiado, cuestión en la cual los técnicos habían trabajado 
para "sensibilizar". De los agricultores familiares entrevistados, 11 plantearon que ahora su práctica productiva de elaboración era más cómoda, uno que realizaba más producción, y dos que tenían mejores prácticas. Es decir, estos cambios, en cuanto a buscar comodidad, no era una problemática que estuviera instalada y tampoco lo era (según los técnicos) el tener que separar la producción doméstica de la destinada a la venta. Ambas inquietudes surgieron por medio del vínculo entre técnicos y agricultores familiares.

Por ello, en referencia a los recursos externos, es importante plantear lo dicho por los técnicos en relación a que el rol del Estado, durante la implementación de este proyecto, fue complejo. Por un lado el Estado debe asegurar la salud de su población general. Sin embargo, no posee categorías o herramientas que le permitan evaluar lo complejo de la unidad familiar de producción. Las herramientas de evaluación (es decir, el Código Alimentario Argentino) no corresponden ni al tipo ni a la escala de producción (lo industrial aplicado a lo familiar), mientras que los ministerios tampoco poseen canales adecuados para intervenir sobre los agricultores familiares, porque las categorías existentes tienden a invisibilizarlos. El Estado no poseía (y en buena medida no posee) programas que se adecuen a la realidad de los agricultores familiares. Si eran concebidos como pobres, el Ministerio de Desarrollo Social era el que debía intervenir, pero éste no financiaba construcción de salas de elaboración (teniendo en cuenta su fin productivo). Si eran pensados como productores, el Ministerio de Agricultura, Ganadería y Pesca debía actuar, pero el volumen a comercializar no parecía llegar a justificarlo. Uno de los técnicos se refiere al tema expresando que "es un problema que tiene la legislación, porque no contempla, o contempla en forma muy laxa o con mucha deficiencia, todo lo que es la producción que está entre lo domestico y lo industrial". Y otro remarca que "los programas no contemplaban la comercialización de la producción. No financiaban la comercialización. Era todo con ese concepto de aquellos años de puertas adentro. La pobreza puertas adentro". Así, el Estado puede funcionar como facilitador u obstáculo, según la circunstancia.

El presente trabajo ha permitido reflexionar sobre la articulación entre proyectos de desarrollo rural orientados a la agricultura familiar, acompañamiento técnico y procesos de planificación vertical desde el Estado a partir del estudio de un proyecto de Salas de Elaboración implementado en la provincia de Misiones. Del estudio realizado surgen propuestas tendientes a repensar la relación entre estos actores para que la aplicación de proyectos responda mejor a las necesidades de los agricultores familiares, facilite el rol de mediación de los extensionistas y se corresponda con las políticas públicas. Partiendo del caso analizado queda clara la necesidad de continuar investigando para profundizar en estas temáticas desde una perspectiva psicosocial.

\section{REFERENCIAS}

ALIAGA, L. et al. Requisitos para habilitar establecimientos de elaboración de alimentos. Allen, Argentina: INTA, 2011.

BOAS, A. A.; GOLDEY, P. A comparison on farmers' participation in farmers' organizations and implications for rural extension in Minas Gerais. Organizações Rurais \& Agroindustriais, v. 7, n. 3, p. 259-270, 2005.

BURNETT, A. As raízes rurais da feira da Sulanca no agreste Pernambucano. Extensão Rural. v. 21, n. 4, p. 9-31, 2014. 
BUSSO, M. Las ferias comerciales: también un espacio de trabajo y socialización. Aportes para su estudio. Trabajo y Sociedad. n. 16, p. 105-123, 2011.

CARBALLO, C. Extensión y transferencia de tecnología en el sector agrario Argentino. Buenos Aires: Universidad de Buenos Aires, 2002.

et al. Perfil y expectativas de los consumidores de las ferias francas de Posadas. Buenos Aires, Facultad de Agronomía, Universidad de Buenos Aires, 2008. (Convenio Proyecto G 019).

DI PIETRO, S. et al. Vigilancia epidemiológica de enfermedades transmitidas por alimentos en la provincia de Río Negro, Argentina, 1993-2001. Medicina (Buenos Aires). v. 64, n. 2, p. 120-124, 2004.

FERNÁNDEZ, P. R.; PICCIARELLI, A. Control de calidad microbiológica de leche y quesos extendidos por pequeños productores. En: Jornada de Iniciación en la Investigación y en la Innovación, 4, 2012, Posadas. Anais..., Posadas, Argentina: CEDIT, 2012.

FREITAS, L. A.; KARAM, K. F.; PINHEIRO, S. L. Construção participativa de arranjos silvipastoris: Um estudo de caso no município de São Bonifácio - SC. Extensão Rural. n. 16, p. 37-66, 2008.

GARCÍA, L. Resistencias campesinas: La experiencia de las ferias francas de la provincia de Misiones, Argentina. En: FERNANDES, B. M. Campesinado y agronegocio. São Paulo: CLACSO, 2008.

; WAHREN, J. Identidades en construcción y acción colectiva de los jóvenes del norte argentino. Una comparación de los casos de la Unión de Jóvenes Feriantes de Misiones y los jóvenes de la UTD de Gral. Mosconi (Salta). En: JORNADAS DE JÓVENES INVESTIGADORES DEL INSTITUTO DE INVESTIGACIONES GINO GERMANI, 3, 2005, Buenos Aires, Anais..., Buenos Aires: Universidad de Buenos Aires, 2005.

GOLSBERG, C. et al. Agricultura familiar: ferias de la agricultura familiar en la Argentina. Buenos Aires: INTA, 2010.

LANDINI, F. P. Prácticas cooperativas en campesinos formoseños. Problemas y alternativas. Revista de la Facultad de Agronomía. v. 27, n. 2, p. 173-186, 2007.

. Racionalidad económica campesina. Mundo Agrario. v. 12, n. 23 [sin páginas], 2011.2 Disponible en: <http://www.scielo.org.ar/pdf/magr/v12n23/v12n23a14.pdf>. Acceso en: 15 enero 2016.

La noción de psicología rural y sus desafíos en el contexto latinoamericano.

En: LANDINI, F. P. Hacia una psicología rural latinoamericana. Buenos Aires: CLACSO, 2015.

Problemas de la extensión rural en América Latina. Perfiles Latinoamericanos. v. 24, n. 47, p. 47-68, 2016 
et al. Towards a psychology of rural development processes and Interventions. Journal of Community and Applied Social Psychology. v. 24, n. 6, p. 534-546, 2014a.

et al. Theoretical guidelines for a psychology of rural development. Cuadernos de Desarrollo Rural. v. 11, n. 74, p. 125-147, 2014b.

; MURTAGH, S.; LACANNA, M.C. Aportes y reflexiones desde la psicología al trabajo de extensión con pequeños productores. Formosa, Argentina: INTA, 2009.

LONG, N.E. Sociología del desarrollo. Una perspectiva centrada en el actor. México: CIESAS, 2007.

Acercando las fronteras entre la antropología y la psicología para comprender las dinámicas de desarrollo rural. En: LANDINI, F. P. Hacia una Psicología Rural Latinoamericana. Buenos Aires: CLACSO, 2015.

MARZOCCA, M. A.; MARUCCI, M. G.; ÁLVAREZ, E. E. Detección de Listeria monocytogenes en distintos productos alimenticios y en muestras ambientales de una amplia cadena de supermercados de la ciudad de Bahía Blanca (Argentina). Revista Argentina de Microbiología. v. 36, n. 4, p. 179-181, 2004.

MICCIO, L. et al. Contaminación de carne molida con cepas de Escherichia coli shigatoxigénico (STEC) provenientes de comercios minoristas de San Martín, Buenos Aires, categorizados según nivel socioeconómico. InVet. v. 13, n. 1, p. 3744, 2011.

MONTERO, I.; LEÓN, O. G. Sistema de clasificación del método en los informes de investigación en psicología. International Journal of Clinical and Health Psychology. v .5, n. 1, p. 115-127, 2005.

OLIVERA-CARRION, M. Criterios de evaluación bromatológica y nutricional de productos pre-elaborados a partir del etiquetado e información técnica. Diaeta. v. 29, n. 137, p. 14-22, 2011.

PROCHASKA, J. O.; NORCROSS, J. C.; DICLEMENTE, C. C. Changing for good A revolutionary six-stage program for overcoming bad habits and moving your life positively forward. Nueva York: William Morrow \& Co, 1994.

MOREL, A. P. S.; REZENDE, L. T.; SETTE, R. S. Negócio feira livre: análise e discussão sob a perspectiva do feirante. Extensão Rural. v. 22, n. 4, p. 43-57, 2015.

RODRÍGUEZ, F. et al. La Feria Franca de San Vicente (Misiones) y sus efectos en la organización interna de las unidades familiares de producción. En: CITTADINI, R. Economía social y agricultura familiar: Hacia la construcción de nuevos paradigmas de intervención. Buenos Aires: INTA, 2010.

ROSENFELD, V.; KRIEGER, C.; CAZZANIGA, H. La agricultura familiar en Misiones: confrontación de modelos de desarrollo. Buenos Aires: Instituto de Desarrollo social y Promoción Humana (INDES), 2005. 
ROTH, E. Cambio social: factores psicológicos asociados a la disposición a cambiar. 2009. 421f. Tesis Doctoral, Universidad de Granada, España.

SARAIVA, R. M.; CALLOU, A. B. F. Políticas públicas e estratégias de comunicação para o desenvolvimento local de comunidades pesqueiras de Pernambuco. Interações. v. 10, n. 1, p. 73-81, 2009

SCHVORER, E. L. La feria franca de Eldorado. Alternativas de desarrollo para la producción agrícola familiar en Misiones. En: HOCSMAN, L. D. Transformaciones productivas e impactos sociales agrarios en años de neoliberalismo. Villa María, Argentina: Universidad Nacional de Villa María, 2007.

SIQUEIRA, H. M. et al. Comercialização solidária da produção familiar de alimentos em Alegre-ES. Extensão Rural. v. 20, n. 3, p. 98-118, 2013.

TAVEIRA, L. R. S.; OLIVEIRA, J. T. A. A extensão rural na perspectiva de agricultores assentados do Pontal do Paranapanema. Revista de Economia e Sociologia Rural. v. 46, n. 1, p. 9-30, 2008. 